\title{
Erratum to: Evaluation of droplet digital PCR for characterizing plasmid reference material used for quantifying ammonia oxidizers and denitrifiers
}

Lianhua Dong • Ying Meng • Jing Wang • Yingying Liu

Received: 12 June 2014 / Accepted: 12 June 2014 / Published online: 29 June 2014

(C) Springer-Verlag Berlin Heidelberg 2014

Erratum to: Anal Bioanal Chem

DOI 10.1007/s00216-013-7546-1

The authors would like to call the reader's attention to the fact that unfortunately the A337 probe sequence in table 2 was wrong. Below please find the correct sequence:

\begin{tabular}{l}
\hline FAM-TTCTACTGGTGGTCRCACTACCCCATCAACT- \\
TAMRA
\end{tabular}

The online version of the original article can be found at http://dx.doi.org/ 10.1007/s00216-013-7546-1.

L. Dong $(\bowtie) \cdot$ J. Wang $\cdot$ Y. Liu

National Institute of Metrology, Beijing 100013, China

e-mail: donglh@nim.ac.cn 